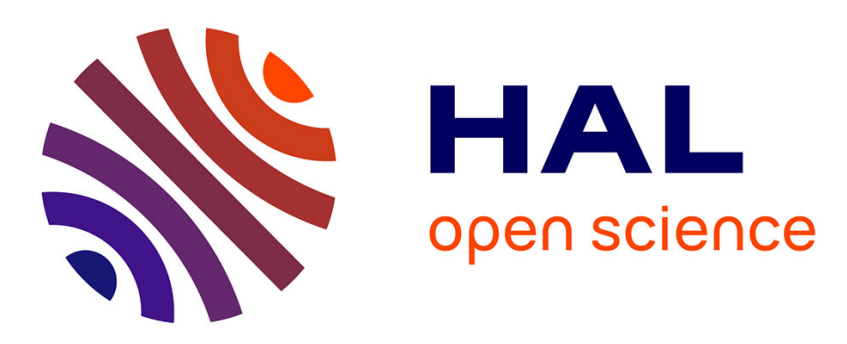

\title{
Example-Based Microstructure Rendering with Constant Storage
}

Beibei Wang, Miloš Hašan, Nicolas Holzschuch, Ling-Qi Yan

\section{To cite this version:}

Beibei Wang, Miloš Hašan, Nicolas Holzschuch, Ling-Qi Yan. Example-Based Microstructure Rendering with Constant Storage. ACM Transactions on Graphics, 2020, 39 (5), pp.1-12. 10.1145/3406836 . hal-03156347

\section{HAL Id: hal-03156347 \\ https://hal.inria.fr/hal-03156347}

Submitted on 2 Mar 2021

HAL is a multi-disciplinary open access archive for the deposit and dissemination of scientific research documents, whether they are published or not. The documents may come from teaching and research institutions in France or abroad, or from public or private research centers.
L'archive ouverte pluridisciplinaire $\mathbf{H A L}$, est destinée au dépôt et à la diffusion de documents scientifiques de niveau recherche, publiés ou non, émanant des établissements d'enseignement et de recherche français ou étrangers, des laboratoires publics ou privés. 


\title{
Example-Based Microstructure Rendering with Constant Storage
}

\author{
BEIBEI WANG, School of Computer Science and Engineering, Nanjing University of Science and Technology, China \\ MILOŠ HAŠAN, Adobe Research, USA \\ NICOLAS HOLZSCHUCH, University Grenoble Alpes, Inria, CNRS, Grenoble INP, LJK, France \\ LINGQI YAN, University of California, Santa Barbara, USA
}
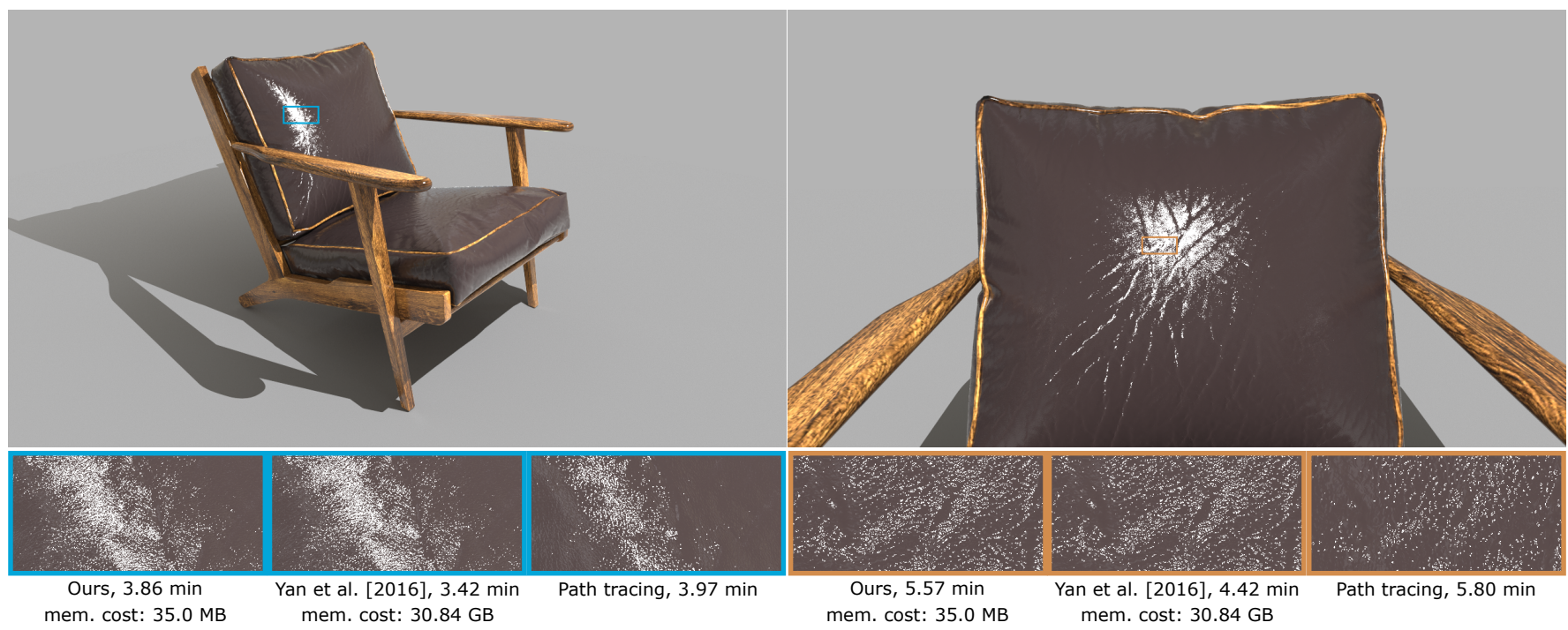

Fig. 1. Our method is capable of rendering detailed specular microstructure like Yan et al. [2016], but with much smaller storage requirements, and without any repetition artifacts. The leather material is represented using two normal maps: a standard macro-level map, and a microstructure map, synthesized on-the-fly using our method from a small $512 \times 512$ example patch. Bottom: zoomed-in images rendered at higher resolution. For Yan et al. we use a $10 \mathrm{~K} \times 10 \mathrm{~K}$ normal map as input. The storage of our method is $35.0 \mathrm{MB}$, while the previous method requires $30.84 \mathrm{~GB}$ to handle a similar level of detail without repetition. For comparison, we also show the rendering using naive path tracing (similar time as our method), where only a fraction of the glints has been found. This fraction would be different in each run, thus the naive approach is unsuitable for animations.

Rendering glinty details from specular microstructure enhances the level of realism, but previous methods require heavy storage for the high-resolution height field or normal map and associated acceleration structures. In this paper, we aim at dynamically generating theoretically infinite microstructure, preventing obvious tiling artifacts, while achieving constant storage cost. Unlike traditional texture synthesis, our method supports arbitrary point and range queries, and is essentially generating the microstructure implicitly. Our method fits the widely used microfacet rendering framework with multiple

Authors' addresses: Beibei Wang, School of Computer Science and Engineering, Nanjing University of Science and Technology, 200 Xiaolingwei Rd, Nanjing, 210094, China, beibei.wang@njust.edu.cn; Miloš Hašan, Adobe Research, San Jose, USA, milos.hasan@ gmail.com; Nicolas Holzschuch, University Grenoble Alpes, Inria, CNRS, Grenoble INP LJK, 655, avenue de l'Europe, Grenoble, France, Nicolas.Holzschuch@inria.fr; LingQi Yan, University of California, Santa Barbara, 2119 Harold Frank Hall, Santa Barbara, CA, 93106, USA, lingqi@cs.ucsb.edu.

Permission to make digital or hard copies of all or part of this work for personal or classroom use is granted without fee provided that copies are not made or distributed for profit or commercial advantage and that copies bear this notice and the full citation on the first page. Copyrights for components of this work owned by others than ACM must be honored. Abstracting with credit is permitted. To copy otherwise, or republish, to post on servers or to redistribute to lists, requires prior specific permission and/or a fee. Request permissions from permissions@acm.org.

(C) 2021 Association for Computing Machinery.

0730-0301/2021/3-ART \$15.00

https://doi.org/10.1145/nnnnnnn.nnnnnnn importance sampling (MIS), replacing the commonly used microfacet normal distribution functions (NDFs) like GGX by a detailed local solution, with a small amount of runtime performance overhead.

CCS Concepts: • Computing methodologies $\rightarrow$ Rendering; Reflectance modeling.

Additional Key Words and Phrases: Rendering, surface microstructure, glints, constant storage, procedural by-example noise

\section{ACM Reference Format:}

Beibei Wang, Miloš Hašan, Nicolas Holzschuch, and LingQi Yan. 2021. ExampleBased Microstructure Rendering with Constant Storage. ACM Trans. Graph. 1, 1 (March 2021), 12 pages. https://doi.org/10.1145/nnnnnnn.nnnnnnn

\section{INTRODUCTION}

Microstructure rendering of glinty details [Yan et al. 2014] has brought a new level of realism to rendering specular highlights, a core effect in computer graphics. This method and subsequent work uses high-resolution normal maps to explicitly define every microfacet normal. However, very large normal maps are required to cover enough surface area without obvious repetition. For example, Yan et al. [2018] used a resolution of one micron per texel, which 
requires a $10 K \times 10 K$ normal map to cover just one square centimeter. Worse, hierarchical acceleration structures over these normal maps are needed for efficient pruning of non-contributing normals, making the storage problem even more severe. Designing normal maps of that size, which moreover need to allow for seamless tiling, requires additional tedious effort. These are key issues reducing the practicality of the prior solutions.

Although texture synthesis methods are ubiquitous, few of them are suitable for the microstructure rendering task. Earlier image quilting techniques [Efros and Freeman 2001; Efros and Leung 1999; Wei and Levoy 2000] and recent neural texture synthesis methods [Gatys et al. 2015; Jetchev et al. 2016; Zhou et al. 2018] are synthesizing an image starting from a core example image. The problem is that, if we would like to query the synthesized image say at index $(100 K, 100 K)$, the method has to actually synthesize the image all the way up to that point, which is a clear violation of our constant storage need. A dynamic point query (giving the exact value anywhere on the synthesized image in constant time) is needed instead.

On the other hand, procedural noise methods, such as Perlin noise [Perlin 1985], Gabor noise [Lagae et al. 2009] and texton noise [Galerne et al. 2017], use a few parameters to control the appearance of a non-repeating noise function over an infinitely large space. By-example noise methods [Galerne et al. 2012; Gilet et al. 2012; Heitz and Neyret 2018] offer more artist controllability by providing an example texture and blending patches from it at different querying positions. These methods require no additional storage, and support on-the-fly point queries. However, for microstructure rendering, we need not only a normal map, but also the corresponding acceleration method for pruning non-contributing regions. Unfortunately, none of these methods currently support min-max queries in an arbitrary range. Such a range query capability (returning the range of normals in any region on the synthesized image, again without actually generating it) is a necessary component of the solution to our problem.

We present a method that implicitly generates the normal map along with a range query capability, so that it can directly fit into the microstructure rendering framework of Yan et al. [2016], but with much lower storage requirements. Our method builds upon by-example noise methods to maximize artist controllability, and generates normal maps by blending patches from input examples. We support dynamic point queries and range queries on the implicit normal map generated using any by-example method, as long as the blending operation is monotonically increasing (as will be defined in Sec. 4.5). With our method, we are able to render microstructure with non-repetitive patterns, with constant storage cost and a small performance overhead over previous methods.

\section{RELATED WORK}

In this section, we organize the related work into two basic categories. We first briefly review previous work on microstructure rendering and capture, then introduce related work on texture synthesis and general procedural appearance.

Microstructure rendering. Surface reflectance in computer graphics is typically described using microfacet theory [Torrance and
Sparrow 1967], which uses smooth analytic functions such as Beckmann [Beckmann and Spizzichino 1987] and GGX [Walter et al. 2007] to model the distribution of surface normals. More recently, Yan et al. [2014] introduced the idea of using patch-local normal distribution functions ( $\mathcal{P}-\mathrm{NDF}$ ) to accurately compute the spatially and directionally varying appearance from explicit specular microstructure such as bumps, brushes, scratches and metallic flakes. The microgeometry is defined using extremely high resolution normal maps. Yan et al. [2016] proposed a position-normal distribution method to accelerate computation, which was later extended to handle wave optics effects [Yan et al. 2018]. All these methods share a common problem with storage cost: the microstructures have to be defined at resolutions of $1-10$ microns per texel, which either requires very large textures (and associated acceleration structures) or leads to tiling artifacts.

Since explicit microstructure is costly to store, a series of methods were designed to model specific effects. Jakob et al. [2014] introduce a procedural BRDF that produces glitter effects from implicit mirror flake distributions without explicitly storing the underlying microstructure, but is not extensible to other kinds of microgeometry, such as brushes and scratches. Raymond et al. [2016] model surfaces as the mixture of a base surface and a collection of 1D scratches, later extended by Werner et al. [2017] for wave optics effects and by Velinov et al. [2018] for real-time performance; these methods work well for scratches but do not support other appearances. The method of Zirr and Kaplanyan [2016] dynamically adds micro-level details to a predefined macro-scale BRDF, but is focused on realtime performance, not on accurate simulation of the appearance of a given microgeometry.

Detailed appearance measurement. Several approaches measure real-world samples and use the measured data to render, either directly or indirectly. Dong et al. [2015] used an interferometry device to acquire the microstructure of brushed metal, but they still use statistical reflectance models to fit the measured data for rendering. Other methods [Graham et al. 2013; Nagano et al. 2015; Nam et al. 2016] aim at measuring accurate heightfields; these could be used with glint rendering methods, but seamless extension of the data across larger surface areas remains a problem, which could be addressed by our method.

Texture synthesis. We wish to generate non-repeating appearance, which is also the goal of texture synthesis. Texture synthesis methods can be categorized into three different kinds. The first kind is by expansion: starting from a small texture, they dynamically "grow" a new larger texture. Representative work of this kind ranges from the classic image quilting methods [Efros and Freeman 2001; Efros and Leung 1999; Wei and Levoy 2000] to modern solutions using Generative Adversarial Networks (GANs) [Jetchev et al. 2016; Zhou et al. 2018]. These methods, however, are not applicable to our problem - to query the value at a specific location on the generated texture, the texture has to be actually generated from its original position to the query. This violates our goals of zero dynamic memory consumption and minimum performance overhead.

The second kind of related texture synthesis work is tiling methods, such as Wang tiles [Cohen et al. 2003; Wang 1961]. These methods first create small tiles from the input texture. These tiles are designed to allow seamless stitching to others, and are thus used 


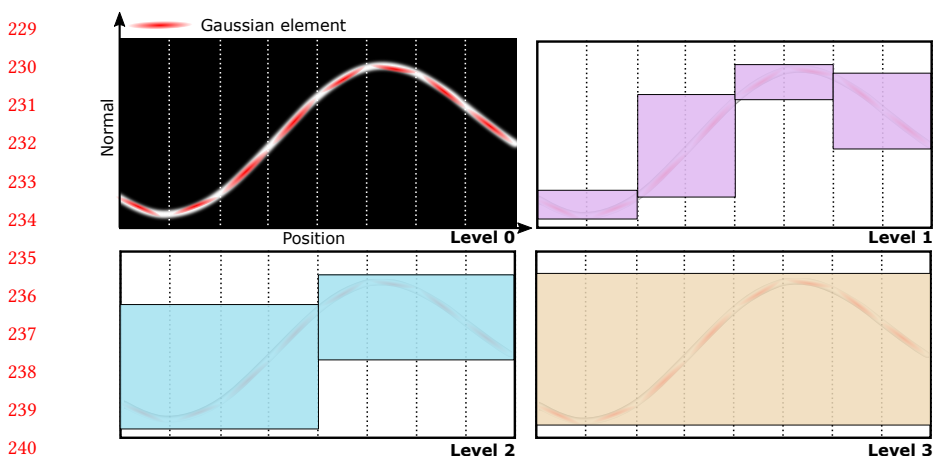

Fig. 2. Top left: Flatland visualization of a position-normal distribution, fitted using Gaussian elements. Each element corresponds to a single texel and its normal. The slope of the element depends on the normal map Jacobian, its horizontal extent matches the texel size, and the vertical thickness is due to intrinsic roughness. Others: Different levels of min-max hierarchy over the normal map bound the sets of normals within spatial ranges.

as building blocks to generate larger textures. The tiling methods can support point queries; however, since the number of tiles is usually limited due to the difficulty of satisfying the seamless tiling property, repeated tiles are often visible as artifacts.

The third kind is blending methods, also known as by-example noise methods. They assume that any point on the resulting texture is blended from several patches from the input texture (example). Different blending methods of the example patches are possible, from simple linear blending (prone to "ghosting" artifacts), to more advanced variance preserving [Yu et al. 2011] and histogram preserving [Heitz and Neyret 2018] methods. These methods are procedural and we demonstrate that they can be adapted to our needs, specifically by designing a suitable point and range query for normals and their Jacobians. Our method does not depend on a specific blending method, and we will show different appearances in Sec. 6 for different choices.

Other procedural appearance. Many efforts have focused on designing procedural noise functions, such as Perlin noise [Perlin 1985] and Gabor noise [Lagae et al. 2009], which give non-repeating values over the entire $2 \mathrm{D}$ or $3 \mathrm{D}$ space. The noise can be later thresholded and post processed in other ways to produce appearance variations that mimic terrain, rust, marble, etc. The noise functions often provide the functionality of point query (the value at any position) and approximate range average query (approximate average value in a given range) for anti-aliasing, but do not support range min-max query (exact minimum and maximum values in a given range), which is a crucial property needed by our method (Sec. 4.3).

\section{BACKGROUND}

\subsection{Rendering details from microstructure}

Our method builds upon the framework of microstructure rendering by Yan et al. [2016], where the microstructure is defined using a high resolution normal map. The normal map is a continuous, differentiable function that returns a $2 \mathrm{D}$ normal $\mathbf{n}(\mathbf{u})=\left(n_{x}, n_{y}\right)$ (dropping the implicit $z$-coordinate) for any given $2 \mathrm{D} \mathbf{u}=(u, v) . \mathrm{A} \mathrm{uv}-$ parameterization on geometric primitives is required to map the normal maps to surfaces. Removing this assumption (so that our method applies to any geometries without need for uv-parameterizations) would be an interesting future work direction.

During rendering, a spatial footprint $\mathcal{P}$ (i.e. coverage on the texture) can be approximated by the renderer as a Gaussian $G \mathcal{P}$; this footprint can be as large as the pixel projection onto the surface, but is typically smaller (leaving some work to pixel multi-sampling). To evaluate the surface BRDF for the footprint $\mathcal{P}$, we need to query the distribution of the surface normals within the footprint, i.e. the patch normal distribution function $(\mathcal{P}-\mathrm{NDF})$. To do that, for every position within $\mathcal{P}$, we check whether its normal is close enough to a query direction $\mathbf{s}$, where the closeness is defined using another Gaussian $G_{r}$ specifying an "intrinsic roughness" of the microstructure. The query can be written formally as

$$
D_{\mathcal{P}}(\mathbf{s})=\int G_{\mathcal{P}}(\mathbf{u}) \mathcal{N}(\mathbf{u}, \mathbf{s}) \mathrm{d} \mathbf{u},
$$

where $\mathcal{N}(\mathbf{u}, \mathbf{s})=G_{r}(\mathbf{n}(\mathbf{u})-\mathbf{s})$ is a $4 \mathrm{D}$ function of $\mathbf{u}$ and $\mathbf{s}$ called position-normal distribution, where $\mathbf{u}$ represents the texture coordinate (surface position), and s represents the normal. Fig. 2 illustrates the position-normal distribution in a simplified flatland case (1D position, 1D normal). Using this definition, the resulting $D_{\mathcal{P}}$ becomes a replacement of the smooth NDF in the classic microfacet model.

Since the $4 \mathrm{D}$ position-normal distribution $\mathcal{N}(\mathbf{u}, \mathbf{s})$ is complicated, Yan et al.[2016] approximate it with a mixture of $k$ Gaussian elements in $4 \mathrm{D}$, such that $\mathcal{N}(\mathbf{u}, \mathbf{s}) \approx \sum_{i=1}^{k} G_{i}(\mathbf{u}, \mathbf{s})$. Each Gaussian element is defined as

$$
G_{i}(\mathbf{u}, \mathbf{s})=c_{i} \exp \left(-\frac{1}{2}\left(\mathbf{x}-\mathbf{x}_{i}\right)^{T} \Sigma_{i}^{-1}\left(\mathbf{x}-\mathbf{x}_{i}\right)\right),
$$

where $c_{i}$ is a constant for normalization, $\mathbf{x}=(\mathbf{u}, \mathbf{s})^{T}$ is a $4 \mathrm{D}$ column vector, and $\Sigma$ is the covariance matrix computed from the Jacobian of the normal $\mathbf{n}(\mathbf{u})$.

The query of the $\mathcal{P}-\mathrm{NDF}$ at $\mathbf{s}$ thus becomes

$$
D_{\mathcal{P}}(\mathbf{s}) \approx \sum_{i=1}^{k} \int G \mathcal{P}(\mathbf{u}) G_{i}(\mathbf{u}, \mathbf{s}) \mathrm{d} \mathbf{u},
$$

where each term of the sum has been simplified to calculating the product integral of two 2D Gaussians (since the two dimensions of $\mathbf{s}$ are given as a query and are constant with respect to integration), which results in an analytical solution.

In theory, Gaussians have infinite support, so every term in the above sum contributes a non-zero quantity. In practice, though, we can truncate the Gaussians, thus introducing small but perceptually negligible error. In the following, we assume truncation at 3 standard deviations. Thus any Gaussian footprint query can be bounded by a square query.

As shown by Yan et al. [2016], converting each texel of a well sampled normal map to a single Gaussian element typically gives good results in practice. Therefore, the number of Gaussian elements $k$ is usually in the millions. To avoid calculating every Gaussian 
element's contribution to every query, Yan et al. [2014] build a minmax hierarchy over the normal map. The hierarchy is a tree structure, where each node stores the range of normals in its child nodes. With the hierarchy, a group of Gaussian elements can be pruned together if the bounding box of the normals is far from the query $\mathbf{s}$, meaning its contribution is negligible, given the Gaussian truncation approach mentioned above. The idea was later extended [Yan et al 2016] to a $4 \mathrm{D}$ acceleration structure over both positions and normals, which is essentially multiple hierarchies for the Gaussian elements contributing to certain ranges of normals.

\subsection{Procedural by-example noise}

The key idea of by-example noise generation is to create a new image patch by blending multiple patches, picked up from different places on a given example. To make this process procedural, at any place on the synthesized noise, we need to know which patches are selected to blend. This is usually done by partitioning the infinite planar domain into regular regions (triangles, quads, etc.), where each region is associated with a unique random seed that is used to pick random patches from the example. Within each region, the blending weights vary linearly. We will describe these weights along with our choice of regions in more detail in Sec. 4.2.

During the noise generation, differences emerge in different choices of patch blending methods. Here, we introduce three representative methods: linear blending, variance preserving blending [Yu et al. 2011], and histogram preserving blending [Heitz and Neyret 2018].

Linear blending is the most straightforward. It is a simple weighted average of all $K$ inputs:

$$
I_{l}=\sum_{i=1}^{K} w_{i} I_{i},
$$

where $w_{i}$ is the weight of the $i$-th input $I_{i}$ at a specific position.

Variance preserving blending builds on top of the linear blending:

$$
I_{v}=\left(I_{l}-\bar{I}\right) / W+\bar{I},
$$

where $W=\sqrt{\sum_{i=1}^{K} w_{i}^{2}}$ is the L2-norm of all the weights, and $\bar{I}$ is the (uniform-weighted) average of all the inputs. Histogram preserving blending considers an additional operation and its inverse to the variance preserving blending. That is, it computes a mapping $\mathcal{G}$ that maps the histogram of the example into a 1D Gaussian distribution. There are three steps: apply the mapping $\mathcal{G}$ to standardize (transform into a standard normal distribution) the example, then perform variance preserving blending, and finally apply the inverse mapping of $\mathcal{G}$ to obtain the blended result. This can be written as

$$
I_{h}=\mathcal{G}^{-1}\left[\mathcal{G}(I)_{v}\right] .
$$

Determining which of these blending methods gives the best visual effects in which scenarios is beyond the scope of our paper. Our method works with all blending methods, as long as they satisfy a monotonicity property, as will be analyzed in Sec. 4.3.

\section{PROCEDURAL MICROSTRUCTURE RENDERING}

In this section, after some definitions, we first describe how to query the infinite synthesized microstructure at a given point; this follows previous by-example synthesis methods, with modifications

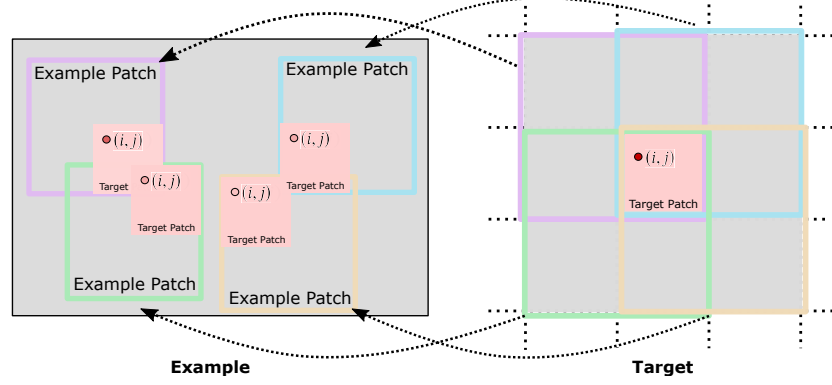

Fig. 3. The target patch (pink square) is the blended result of four different example patches (squares with different color). Each example patch has a deterministic random location in the example, which is specified by the target patch index. Each point in the target patch has one blending weight (represented as opacity of the small red dot, where higher opacity represents more weight) for each blending example patch. These blending weights vary across the target patch.

for querying normals and their Jacobians. Second, we describe our approach to query the range of normals that occurs within a given square range of the infinite microstructure; this is a key innovation over previous work, based on the range minimum query (RMQ) data structure. Third, we show how to use this range query to isolate contributing texels within a given footprint.

\subsection{Definitions}

Before we proceed, we first define the terminology we are going to use:

- example - the given input normal map (not necessarily tileable),

- example patch - a square patch from the example,

- target - the infinitely large planar domain on which we synthesize the microstructure, and

- target patch - a square patch on the target that is formed by blending several example patches.

We specify our goals formally as:

(1) point query - given a texture coordinate $\mathbf{u} \in[-\infty, \infty]^{2}$, query the normal map value $\mathbf{n}(\mathbf{u})$ and its Jacobian $\mathbf{J}(\mathbf{u})$ of the implicitly synthesized microstructure at a time complexity of $O(1)$, and

(2) range query - given a square target patch $\left[\mathbf{u}_{1}, \mathbf{u}_{2}\right]$ (top-left and bottom right corners), query the interval that tightly bounds the values within the patch, $\left[\mathbf{n}_{x}^{\min }, \mathbf{n}_{x}^{\max }, \mathbf{n}_{y}^{\min }, \mathbf{n}_{y}^{\max }\right]$, also at a time complexity of $O(1)$.

In the next subsections, we will introduce the point query and the range query, then describe how these two operations are used together for fast $\mathcal{P}$-NDF queries during the rendering process.

\subsection{Point query}

The point query operation consists of two different parts. First, based on the point query's location, it finds the target patch it stays in and the corresponding example patches. Second, it performs blending from different points on different example patches. These parts are 
similar to the by-example texture synthesis methods introduced in Sec. 3, with some modifications.

As Fig. 3 shows, the target is covered by overlapping example patches. The example patches are squares, and they overlap each other by half the edge length. Thus, any target patch is the blended result of four different example patches. The blending weights are bilinearly interpolated within the patch.

We partition the target into a square grid of target patches. Each target grid vertex is assigned a random number (seed) computed by hashing its index $(i, j)$. This random number is used to locate a specific example patch. Based on the relative position of the point query inside the target patch, we immediately know which four example patches are being blended at this point.

The second step is to get the value $\mathbf{n}$ and its Jacobian $\mathbf{J}$ at $\mathbf{u}$ on the implicitly synthesized microstructure. It is straightforward to calculate the blended normal value $\mathbf{n}$. Since we already know the four positions on the example patches, we can immediately get $\mathbf{n}$ by applying the blending methods using Eqns. 4, 5 and 6 or any other methods.

We also need to compute the blended Jacobian J. One immediate way is to perform the same point query of normals at four adjacent locations, then compute the Jacobian using central finite differences However, this method is slower due to multiple queries, and depends on the fixed step size of the numerical differentiation. Instead, we use a fast and accurate analytic solution, described in the Appendix.

\subsection{Range query}

Suppose a pixel footprint $\mathcal{P}$ (a square bounding the truncated Gaussian) is given on the microstructure. We would like to perform subdivision of the pixel footprint to prune areas with non-contributing normals. Essentially, the pruning scheme needs to answer the question: is the queried normal value contained within the interval of normals of a given patch, i.e. between its minimum and maximum values?

Our insight is that we do not need to explicitly build any hierarchy, as long as we are able to answer the query for minimum and maximum normal values, given any positional range on the implicit microstructure. Since any target range is blended from four patches on the example texture, our range query problem becomes two sub-problems. First, querying the minimum and maximum values on the example texture. Second, computing the combined min-max interval as we blend the four query results from the example.

\subsection{Range minimum query}

The first task is a classic algorithmic problem known as the Range Minimum Query (RMQ). In 1D, the RMQ problem can be solved within $O(1)$ runtime and $O(n \log n)$ precomputation time and storage, using the sparse-table algorithm [Bender and Farach-Colton 2000]. As Fig. 4 shows, the key idea is to precompute the answers to all possible range queries of length $2^{K}$, where $K$ is a positive integer. For a general query from the $i$-th element to the $j$-th element, i.e. one with length different from a power of 2 , it takes constant time to find two precomputed range queries, such that (1) one starts at $i$ and the other ends at $j$, (2) they are of the same length and (3) their union covers the entire range $[i, j]$. Then the minimum of
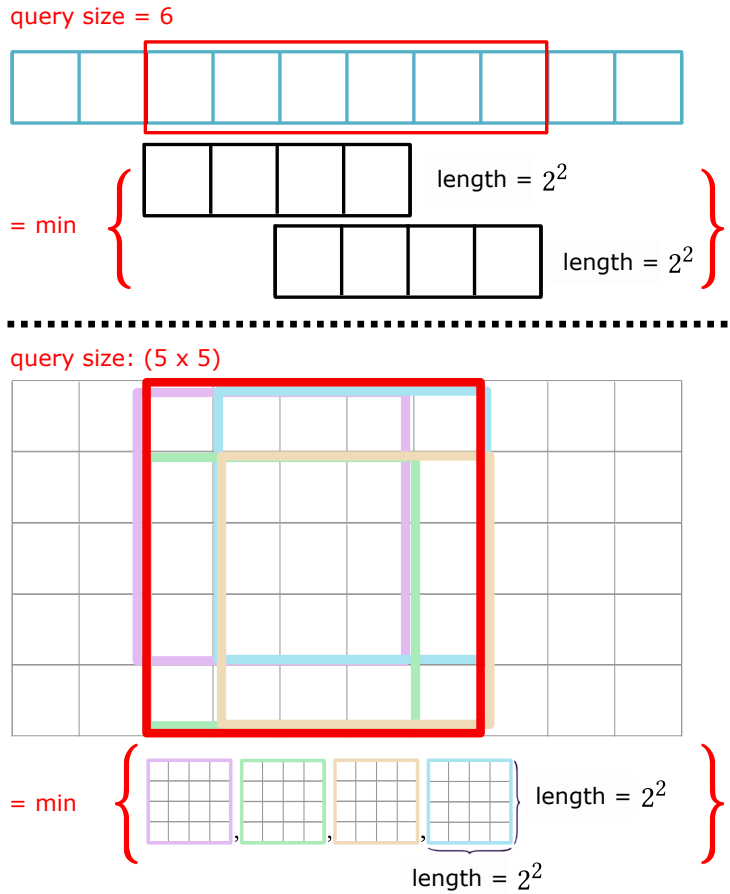

Fig. 4. Top: $1 \mathrm{D}$ version of RMQ. For an arbitrary $1 \mathrm{D}$ query (here, [2,7], marked in red), we first find two precomputed range queries $([2,5]$ and $[4,7])$ with length $2^{2}$; then the minimum of the query $[2,7]$ is the minimum of the two precomputed minima. Bottom: $2 \mathrm{D}$ version of RMQ. For an arbitrary $2 \mathrm{D}$ query (here, $[2,1]$ to $[6,3]$, marked in red rectangle)), we find four precomputed range queries: $[2,1]$ to $[5,2]$ (light purple), $[3,1]$ to [6,2] (light blue), [2,2] to [5,3] (light green) and [3,2] to [6,3] (light yellow), with size $2^{2} \times 2^{1}$. The minimum of the query is the minimum of the four precomputed minima.

the general query $[i, j]$ is the minimum of the two precomputed minima. Note this algorithm gives the exact minimum (not a conservative approximation); the same approach can be used for a range maximum query.

Extending this 1D algorithm to arbitrary 2D queries is straightforward. As illustrated in Fig. 4, we precompute the answers to all possible square range queries of size $2^{K} \times 2^{K}$, where $K$ is a positive integer. For an arbitrary query, we can immediately locate four precomputed range queries of the same size that cover the entire query range, each staying in one of the fours corners of the query range. The minimum of the general query is the minimum of the four precomputed minima. Then the $2 \mathrm{D}$ RMQ problem can be solved within $O(1)$ runtime and $O\left(n^{2} \log n\right)$ precomputation time and storage, where $n \times n$ is the resolution of the $2 \mathrm{D}$ array.

Note that the precomputed data in our 2D sparse-table algorithm is different from a mip-map style tree structure. Taking the 1D case as an example, a precomputed query in a tree structure must start at multiples of its length. However, the sparse-table algorithm precomputes for all possible starting points. The difference indicates why a tree structure only supports range queries within $O(\log n)$ time. 


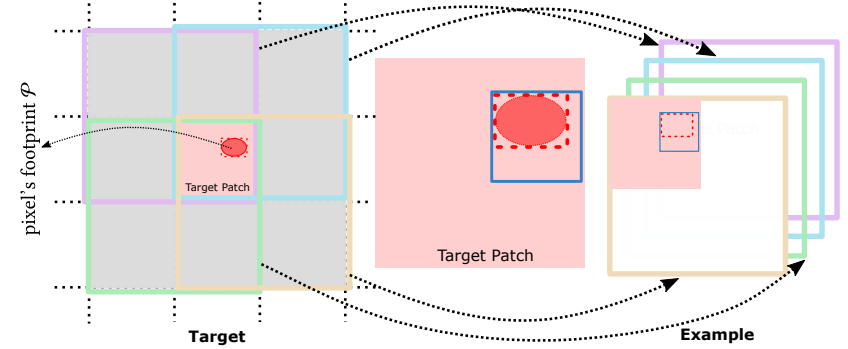

Fig. 5. We perform the range query in the target patch instead of the footprint. We find the tightest square bounding the pixel's footprint $\mathcal{P}$, and start the traversal from the square (blue square).

With these tools, we are able to solve the first sub-problem to perform a range query within each example patch. We list the pseudocode for algorithms that build our optimized 2D sparse-table in Algorithm 1 and the pseudocode to use it for our range queries in Algorithm 2 (see the Appendix). The next step is to combine the four queried min-max intervals into one for the target patch, as the normals within these intervals on the example patches are blended.

\subsection{Blending range queries}

The problem of accurately combining the range queries from the patches being blended still remains. The union of the four queries may not be a conservative bound, since more advanced blending methods may not satisfy the convex-hull property; that is, the blended minimum value could be smaller than any of the input minima.

Our goal is to correctly bound the blended min-max intervals, and make them as tight as possible. To achieve this, we find that all the operations in the by-example noise methods we use, whether linear or non-linear, are monotonically increasing with respect to the values being blended. This property can be easily verified. Elementary operations used in the methods, such as additions/subtractions and multiplications/divisions by positive values, as well as linear operations with positive weights, clearly satisfy the property. The standardization operation in histogram-preserving blending is essentially equivalent to $1 \mathrm{D}$ optimal transport [Monge 1781] (recall that we blend the $x$ and $y$ components of normals separately); therefore, it is also guaranteed to be monotonically increasing [Bonneel et al. 2011].

The monotonically increasing property allows us to apply the same blending method to the endpoints of the min-max intervals from the four source example locations being blended, producing a guaranteed conservative bound. For example, if values $x_{1}, \cdots, x_{4}$ are bounded from above by $u_{1}, \cdots, u_{4}$ respectively, then the blend of the former will be upper-bounded by the same blend of the latter.

However, one additional issue is that the blending weights themselves can vary over the queried range. This means we also need to bound the range of blending weights over a query region. This is straightforward to do within the traversal scheme that uses our range query, which will be introduced in the next subsection. The

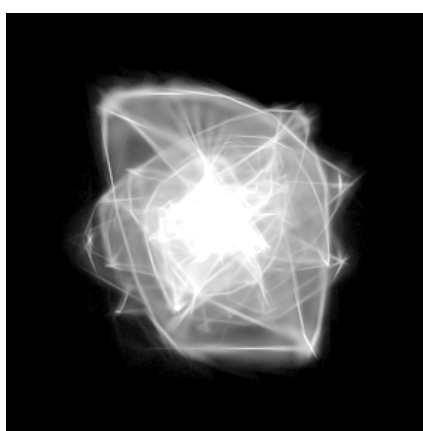

sampling

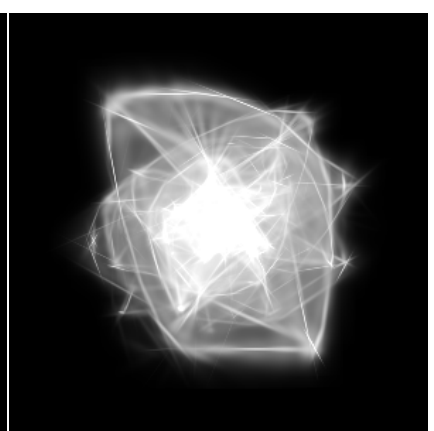

evaluation
Fig. 6. Normal distribution function visualizations with binning the importance sampled directions and with per-pixel evaluation provide closely matching results.

resulting min-max interval of the blended normal is no longer guaranteed to be tight, but is always correct (conservatively bounding) and works efficiently in practice.

\subsection{Implicit hierarchy traversal}

Given the pixel's footprint $\mathcal{P}$, we find its overlap with target patch, and then get the tightest square bounding it, and start the traversal from the square (see Alg. 3). The square is subdivided into smaller squares until their content overlaps entirely with the bounding box (red dash rectangle) of the footprint.

For all these squares, if there are more than one texels, we perform a range query to get their min-max normal interval. If the half vector locates in the normal range, we subdivide the square into four smaller squares and continue the traversal. If the half vector is not included in the range, then all the texels in the square are discarded. The traversal continues until there is only one texel in the square. If the half vector is located in the min-max interval of the texel, we blend the normals and the Jacobians to get a Gaussian element. Finally, we gather the contribution from the Gaussian element.

The min-max interval for the top levels might not be tight in the beginning. However, as the traversal proceeds until lower levels, min-max interval becomes more and more accurate, and converges to the tightest boundary on the finest level. The worst-case time complexity of the query is the same as in previous work [Yan et al. 2016]: it occurs when none of the texels within the footprint are pruned and have to perform the point query; however, this situation is rare.

\section{IMPLEMENTATION DETAILS}

\subsection{Example range precomputation and packing}

Given an example range, the minimum and maximum normals need to be quickly computed. In our implementation, we use a 3D precomputed table to represent the minimum and maximum normal: two dimensions represent corner location in the example, and the third dimension represents the logarithm of query size. The table is computed recursively, starting from the finest level. 
To save memory, we further pack four values (two for minimum normal and two for maximum normal) (the two values are the $\mathrm{x}$ and y components of the normals) into 64 bits. The four values are normalized into $[0,1]$, converted into 16-bit integers and then combined into a 64 bit value. In the end, our precomputed range query table is compact, about $14 \mathrm{MB}$ for a $512 \times 512$ example texture

\subsection{Footprint coverage and multiple target patches}

Each footprint might cover multiple target patches. We bound the footprint within each covering target patch and generate several small sub-footprints. We call these sub-footprints footprints, for simplicity.

\subsection{Importance sampling}

For a given shading point, we find four normals and Jacobians of the four texels around it to get four Gaussian elements. By picking an element proportional to its contribution to the footprint, then picking a normal from that element, we obtain the sampled direction. We validate the correctness of our importance sampling in Figure 6.

\section{RESULTS AND COMPARISON}

We have implemented our algorithm inside the Mitsuba renderer [Jakob 2010]. We compare against Yan et al. [2016] for quality validation. All timings in this section are measured on a $2.20 \mathrm{GHz}$ Intel i7 (images cores) with 32 GB of main memory. Unless otherwise specified, all timings correspond to pictures with $1280 \times 720$ pixels, except the Bent Quad scene with $512 \times 512$. In all of our results, we use histogram preserving blending [Heitz and Neyret 2018] as the blending method, except in Figure 10.

In Table 1, we report all the scene settings, computation time and memory costs for our test scenes. The memory cost in the table is for histogram preserving blending. The other methods (linear and variance preserving blending) cost slightly less than histogram preserving blending.

Chair scene. This scene shows a chair with two leather pillows ( $75 \mathrm{~cm}$ wide), rendered using environment lighting. The leather pillows have a macro-level normal map and detailed microstructure bumps. The macro map covers $75 \mathrm{~cm} \times 75 \mathrm{~cm}$. The micro example normal map with resolution $512 \times 512$ covers $37.5 \mathrm{~mm} \times 37.5 \mathrm{~mm}$ In Yan et al. [2016], we synthesize an equivalent large normal map $(10 K \times 10 K)$ offline and use it for rendering. Compared to Yan et al. [2016], our method produces the same results, with only a fraction $(0.11 \%)$ of memory cost. Regarding the time cost, our method has a small overhead (13\%) over Yan et al. [2016].

Laptop. This scene shows a laptop with a roughened aluminum matte finish. It is rendered using a point light and environment lighting. The laptop is about $30 \mathrm{~cm}$ wide. The input example $512 \times 512$ covers $3 \mathrm{~mm} \times 3 \mathrm{~mm}$. In Yan et al. [2016], we use the same small input texture and tile it. In Figure 9, we can observe obvious repeating patterns in the results of Yan et al. [2016]. In Table 1, we report the memory cost of both methods. Our method costs $35 \mathrm{MB}$, while Yan et al. [2016] costs $62 \mathrm{MB}$ for the same (small) normal map, as our range query tables are slightly more space-efficient than their hierarchy.

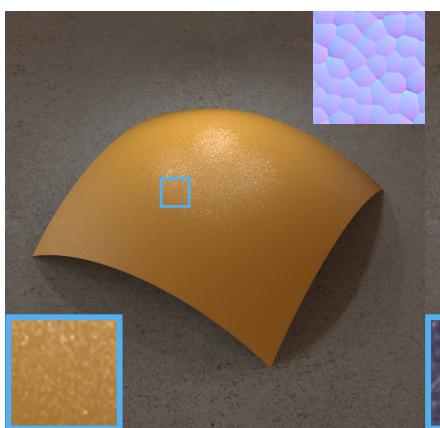

(a) orange plastic, spherical bumps

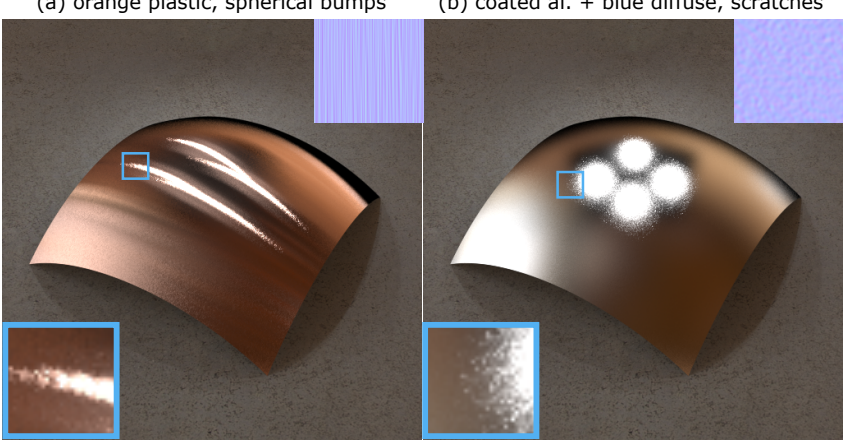

(c) copper, brushed metal (d) al., isotropic noise

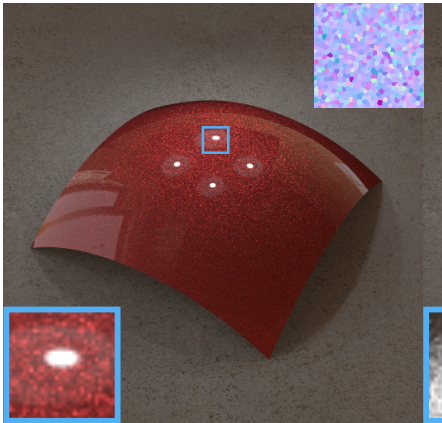

(e) coated al. + red diffuse, metallic flakes

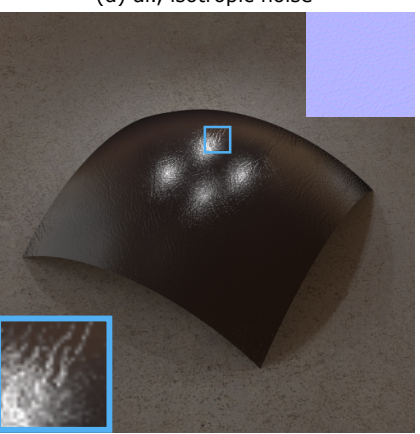

(f) brown plastic, leather

Fig. 7. Rendered results of different normal maps on the Bent Quad scene.

In Figure 10, we compare the results of our method with different blending methods: linear, variance preserving and histogram preserving blending. Our method does not rely on any specific blending method. We observe both variance preserving and histogram preserving blending provide acceptable quality.

In Figure 11, we compare the histogram preserving blending method used in our method with Wang tiles on the Laptop scene. We used the ASTex library for the Wang tiles implementation. We set the tile size as $128 \times 128$ with 16 tiles. The Wang tiles method yields obvious structured artifacts, due to the replication of a small number of tiles; the randomized distribution of the tiles is not sufficient to hide the pattern.

Kettle scene. Figure 8 illustrates a Kettle with brushed metal on the body under two small area lights and environment lighting. The kettle is about $30 \mathrm{~cm}$ high. The input brushed metal normal map with $512 \times 512$ resolution covers about $9 \mathrm{~mm} \times 9 \mathrm{~mm}$. For Yan et 


\begin{tabular}{|c|c|c|c|cc|cc|cc|c|c|}
\hline Scene & \#Tri. & Intr. Rough. & Spp. & \multicolumn{2}{|c|}{ Normal map (ours) } & \multicolumn{2}{|c|}{ Normal map (Yan[2016]) } & \multicolumn{2}{|c|}{ Time (min.) } & Memory (MB) \\
\hline & K & & & Res. & Tile & Res. & Tile & Ours & Yan[2016] & Ours & Yan[2016] \\
\hline Chair & 303.0 & 0.01 & 1024 & $512^{2}$ & 10 & $10 K^{2}$ & 1 & 3.86 & 3.42 & 35.0 & 31584.0 \\
\hline Laptop & 18.4 & 0.005 & 1024 & $512^{2}$ & 100 & $512^{2}$ & 100 & 6.71 & 4.38 & 35.0 & 62.0 \\
\hline Kettle & 175.3 & 0.005 & 1024 & $512^{2}$ & 32 & $2 K^{2}$ & 8 & 3.90 & 3.50 & 35.0 & 1119.9 \\
\hline BentQuad & 19.6 & 0.005 & 1024 & $1 K^{2}$ & 2 & $1 K^{2}$ & 2 & 1.26 & - & 148.0 \\
\hline Shoe & 13.3 & 0.01 & 1024 & $512^{2}$ & 20 & $512^{2}$ & 20 & 3.43 & 3.33 & 35.0 & 62.0 \\
\hline
\end{tabular}

Table 1. Scene settings, computation time and memory costs for our test scenes. \#Tri. is the count of triangles in the scene. Intr. Rough. presents the intrinsic roughness of the material. Spp. represents sample per pixel for path tracing. Normal map (ours) and Normal map (Yan[2016]) represent the input normal map setting for our method and Yan et al. [2016]. The memory cost of our method includes the RMQ table cost, precomputed standardization cost and the input normal map cost.
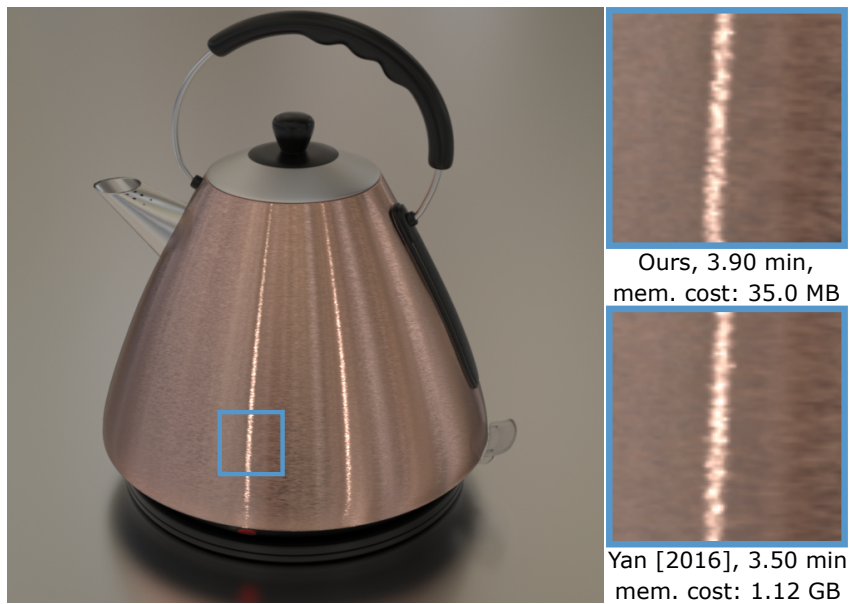

Ours, $3.90 \mathrm{~min}$, mem. cost: $35.0 \mathrm{MB}$

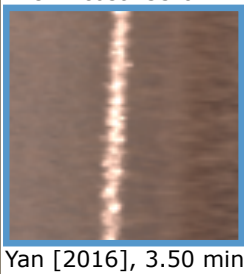

Yan [2016], $3.50 \mathrm{~min}$ mem. cost: $1.12 \mathrm{~GB}$

Fig. 8. Comparison between our method and Yan et al. [2016] with a tiled texture on the Kettle Scene. Normal map: brushed metal. The results are similar, but the memory cost of our method in only a small fraction (about $3 \%$ ) of theirs.

al. [2016], we used a $2 K \times 2 K$ tillable input texture and tiled it. There are no visible differences between our results and those of Yan et al. [2016] (see Figure 8). The memory cost for our method is only a small fraction (about $3 \%$ ) of theirs.

Bent Quad scene. Figures 7 and 12 show a simple scene with a $5 \mathrm{~cm} \times 5 \mathrm{~cm}$ bent quad with a scratched normal map illuminated by a textured light. The resolution of input isotropic noise normal map is $1 K \times 1 K$, and covers $2.5 \mathrm{~cm} \times 2.5 \mathrm{~cm}$. In Figure 12 we show the results with BRDF sampling only, evaluation only and their combination under the multiple importance sampling framework. We also show the result with environment lighting in the right image.

In Figure 7, we show the results of Bent Quad with different BRDF types with different normal maps used as examples.

Shoe scene. This scene shows a shoe with coated metallic flakes under environment lighting. We found that no existing blending method works well with flakes. However, we can easily fix this by using our method without blending (choosing each point from a single patch); the rest of the framework is unchanged. The blended normal maps will have visible seams. However, since every flake has a constant normal, its Jacobian is always zero and does not have to be re-computed from a normal map. Thus, discontinuities in the blended normal maps will not introduce any discontinuous artifacts during rendering. As shown in Figure 13, even though without blending the method produces boundary artifacts in the synthesized normal maps, this problem is not visible in the rendering results.

\section{DISCUSSION AND LIMITATIONS}

Storage overhead breakdown. Figure 14 (left) shows the memory cost of our method and Yan et al. [2016] over varying resolutions. The resolution of the example texture in our method is $512 \times 512$. The memory cost of our method stays consistent with varying texture resolution, while the cost of Yan et al. [2016] increases drastically. In Figure 14 (right), we show the memory cost of components in our method and Yan et al. [2016] for a normal map with resolution $2 K \times 2 K$. The memory cost of our method includes three components: flakes, RMQ precomputed table, normal lookup table and Jacobian lookup table, while the memory cost of Yan et al. [2016] includes two components: flakes and hierarchy.

Our proposed method has several limitations. Our method is under the framework of Yan et al. [2016], so it inherits the limitation of handling only a single reflection, with no multiple scattering or layering. In addition, our method inherits the limitations of the by-example blending methods we use: namely, the restriction to textures with mostly stationary structure, without macroscopic features. Finally, our method does not resolve the problem of increasing computational cost for distant viewing (large footprints), which is also unsolved in previous work. In this case, the number of contributing Gaussian elements to be evaluated becomes large, resulting in expensive queries.

\section{CONCLUSION AND FUTURE WORK}

We have presented a method that allows rendering of specular glints from an arbitrarily large, non-repeating synthesized microstructure. Our method has constant storage cost and a small performance overhead. By designing point query and range query schemes for general by-example texture synthesis methods, we are able to dynamically and implicitly generate an infinite normal map, together with the required Jacobians and range queries. We demonstrate that our method produces plausible and controllable details, supports inputs from any source, and fits into a Monte Carlo rendering framework with multiple importance sampling. Our method can be treated as a standard BRDF, much like the common microfacet 


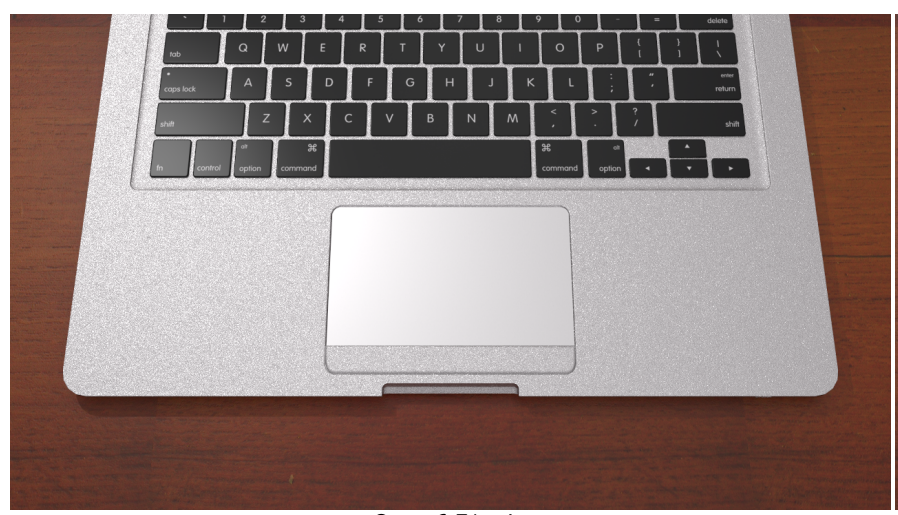

Ours, $6.71 \mathrm{~min}$

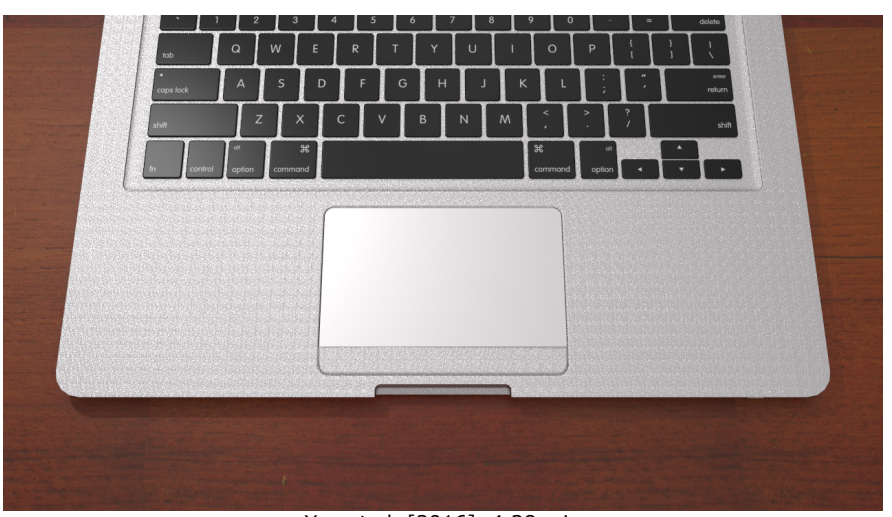

Yan et al. [2016], $4.38 \mathrm{~min}$

Fig. 9. Comparison between our method and Yan et al. [2016] with a tiled texture on the Laptop Scene. The repeated pattern is visible in Yan et al. [2016]. Normal map: isotropic noise.
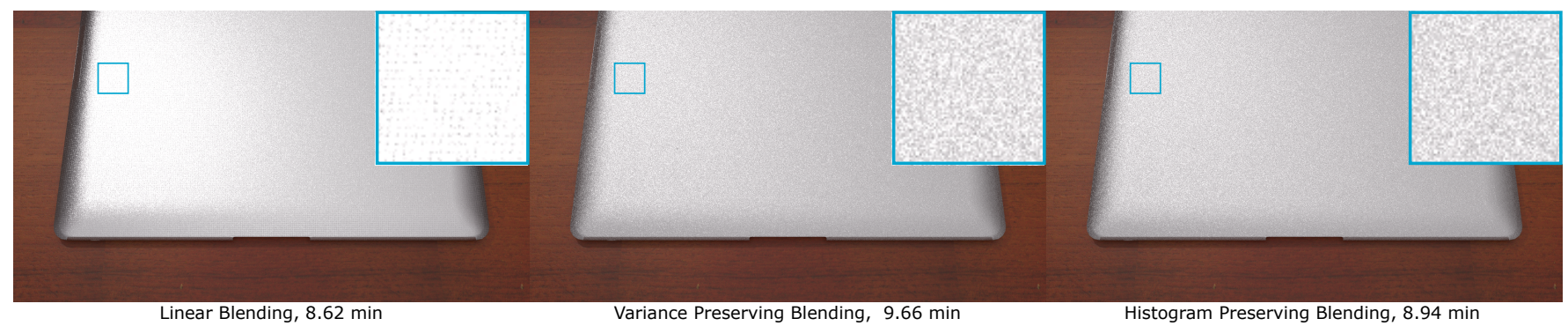

Fig. 10. Comparison between different blending method (linear, variance preserving and histogram preserving on the Laptop Scene. Normal map: isotropic noise. Linear blending has artifacts issues. Both variance preserving and histogram preserving blending provide acceptable quality.

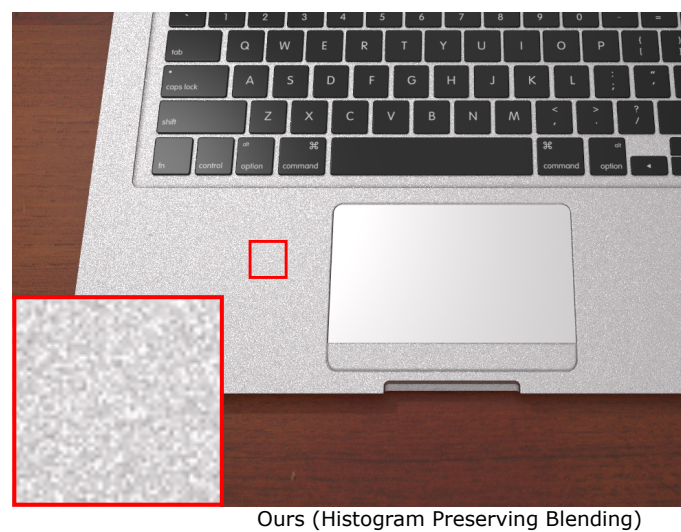

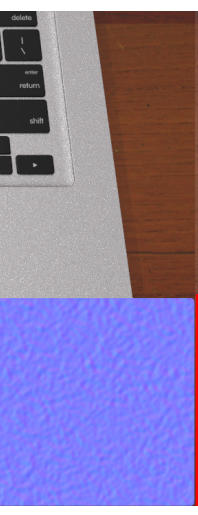
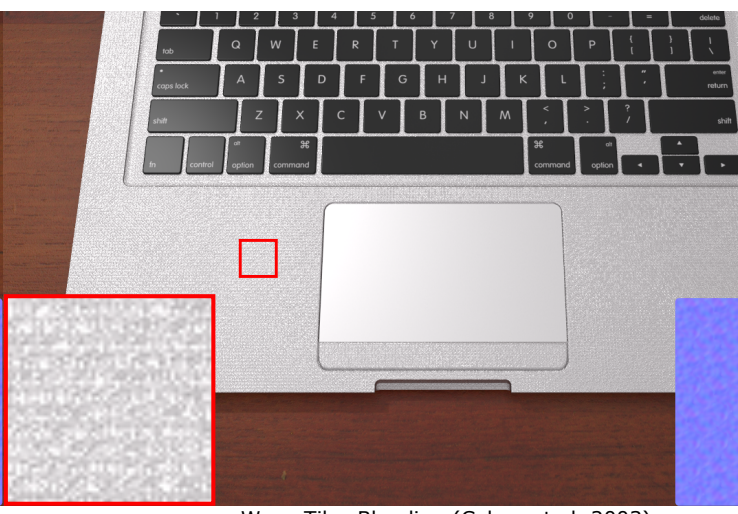

Wang Tiles Blending (Cohen et al. 2003)

Fig. 11. Comparison between our method using histogram preserving blending and Yan et al. [2016] with an offline generated texture (5K $\times 5 K$ with 10 tiles) using Wang tiles [Cohen et al. 2003] on the Laptop scene. The results with Wang tiles suffer from tile artifacts: despite the randomization provided by Wang tiling, the repetition of a small number of base tiles is easily visible to the human eye. Part of the blended normal maps of both histogram preserving blending and Wang tiles are shown at the bottom right.

BRDF [Walter et al. 2007] but replacing the smooth NDF with our solution.

In the future, it would be interesting to optimize our method for real-time implementation on GPUs. Extending our method for rendering with wave optics, or making it work for surfaces without a uv-parameterization, could also be worthwhile directions. 


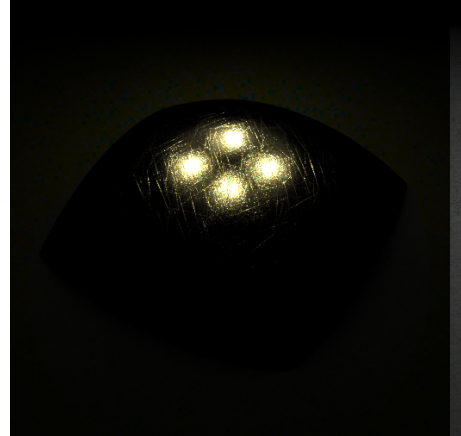

(a) sampling

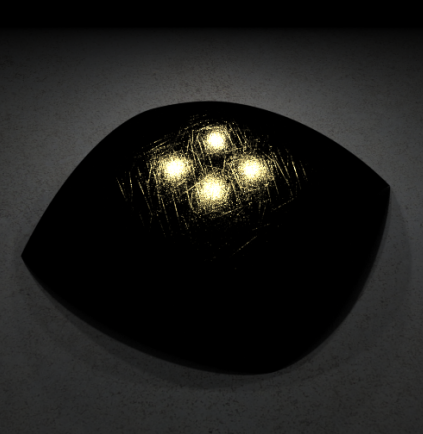

(b) evaluation

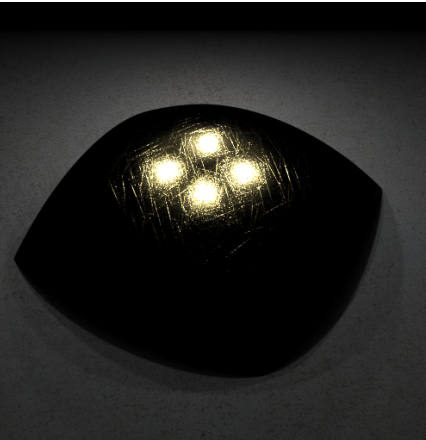

(c) combined

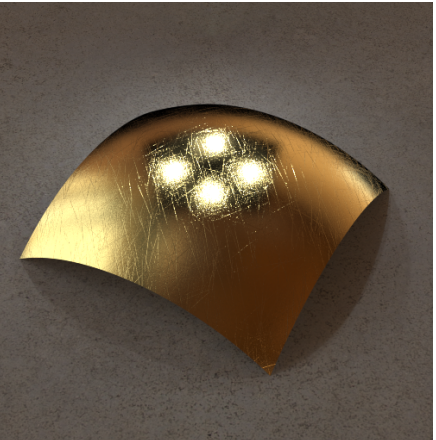

(d) with envmap, 1,26 min

Fig. 12. Our material model can be used inside a standard BRDF sampling/evaluation framework with multiple importance sampling. BRDF sampling alone (a) captures only a small fraction of scratches. Light sampling (b) captures illumination from the high-intensity parts of the HDR light texture onto the scratches. The combined result (c) has the benefits of both estimators. (d) shows the result with extra environment lighting.

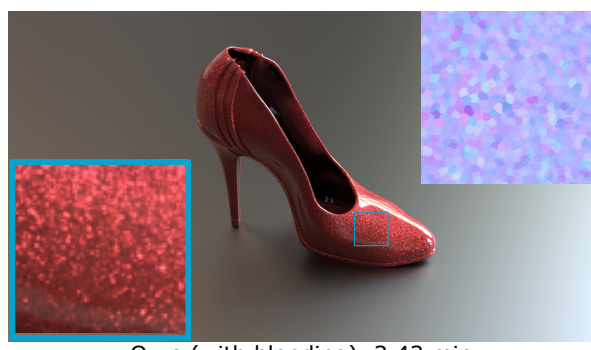

Ours (with blending), 3.43 min

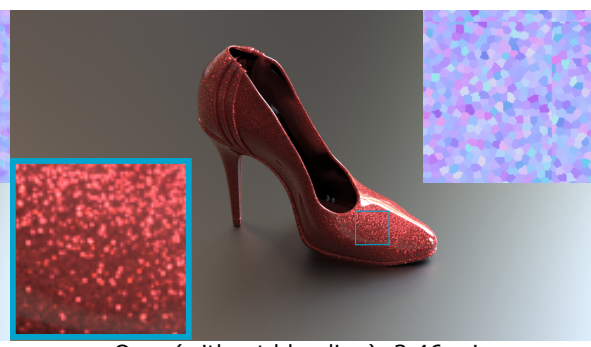

Ours (without blending), 3.46 min

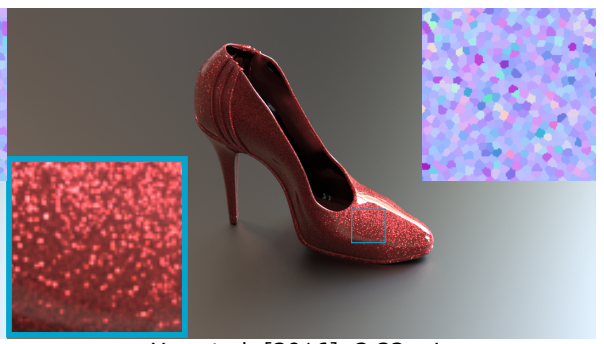

Yan et al. [2016], 3.33 min

Fig. 13. Comparison between our method (with blending), our method (without blending) and Yan et al. [2016] with a tiled texture on the Shoe scene. The normal map depicts metallic flakes as small constant regions. Our method (with blending) has an over-smoothing issue on the metallic flake normal map. However, using synthesis with no blending fixes this issue: while it produces normal maps with boundary artifacts, it does not visually affect the rendering results.
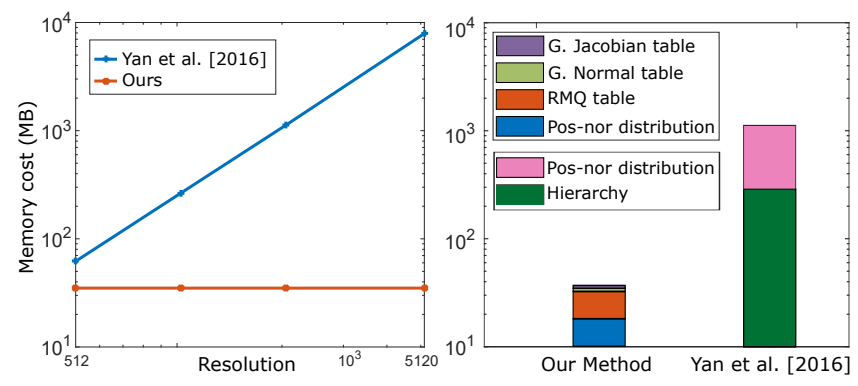

Fig. 14. Left: The memory cost of our method and Yan et al. [2016] over varying texture resolution. The resolution of the example texture in our method is $512 \times 512$. Our method has some overhead, but it its storage needs are only proportional to the example texture. Even when the target resolution is just $512 \times 512$, our method still requires fewer resources than Yan et al., because our RMQ-based solution needs smaller data structures than previously used hierarchies. Right: The memory cost of components in our method and Yan et al. [2016] for resolution $2 K \times 2 K$. G. is short for standardization. Pos-nor is short for position-normal.

\section{REFERENCES}

Petr Beckmann and Andre Spizzichino. 1987. The scattering of electromagnetic waves from rough surfaces. Norwood, MA, Artech House, Inc., 1987, 511 p. (1987).
Michael A. Bender and Martin Farach-Colton. 2000. The LCA Problem Revisited. In Proceedings of the 4th Latin American Symposium on Theoretical Informatics (LATIN '00). 88-94.

Nicolas Bonneel, Michiel van de Panne, Sylvain Paris, and Wolfgang Heidrich. 2011 Displacement Interpolation Using Lagrangian Mass Transport. ACM Transactions on Graphics (Proceedings of SIGGRAPH Asia 2011) 30, 6 (Dec. 2011), 158:1-158:12.

Michael F. Cohen, Jonathan Shade, Stefan Hiller, and Oliver Deussen. 2003. Wang Tiles for Image and Texture Generation. ACM Transactions on Graphics (Proceedings of SIGGRAPH 2013) 22, 3 (2003), 287-294.

Zhao Dong, Bruce Walter, Steve Marschner, and Donald P. Greenberg. 2015. Predicting Appearance from Measured Microgeometry of Metal Surfaces. ACM Transactions on Graphics 35, 1 (2015), 9:1-9:13.

Alexei A. Efros and William T. Freeman. 2001. Image Quilting for Texture Synthesis and Transfer. Proceedings of SIGGRAPH 2001 (August 2001), 341-346.

Alexei A. Efros and Thomas K. Leung. 1999. Texture Synthesis by Non-parametric Sampling. In IEEE International Conference on Computer Vision. Corfu, Greece, 10331038.

Bruno Galerne, Ares Lagae, Sylvain Lefebvre, and George Drettakis. 2012. Gabor Noise by Example. ACM Transactions on Graphics (Proceedings of ACM SIGGRAPH 2012) 31, 4 (2012), 73:1-73:9.

Bruno Galerne, Arthur Leclaire, and Lionel Moisan. 2017. Texton Noise. Computer Graphics Forum 36, 8 (2017), 205-218.

Leon A. Gatys, Alexander S. Ecker, and Matthias Bethge. 2015. A Neural Algorithm of Artistic Style. arXiv:cs.CV/1508.06576

Guillaume Gilet, Jean-Michel Dischler, and Djamchid Ghazanfarpour. 2012. Multiple Kernels Noise for Improved Procedural Texturing. Vis. Comput. 28, 6-8 (2012), 679-689.

Paul Graham, Borom Tunwattanapong, Jay Busch, Xueming Yu, Andrew Jones, Paul Debevec, and Abhijeet Ghosh. 2013. Measurement-based synthesis of facial microgeometry. Computer Graphics Forum 32, 2pt3 (2013), 335-344. 
Eric Heitz and Fabrice Neyret. 2018. High-Performance By-Example Noise Using a Histogram-Preserving Blending Operator. Proc. ACM Comput. Graph. Interact. Tech. 1, 2 (2018), 31:1-31:25

Wenzel Jakob. 2010. Mitsuba Renderer. http://www.mitsuba-renderer.org/.

Wenzel Jakob, Miloš Hašan, Ling-Qi Yan, Jason Lawrence, Ravi Ramamoorthi, and Steve Marschner. 2014. Discrete Stochastic Microfacet Models. ACM Transactions on Graphics (Proceedings of SIGGRAPH 2014) 33, 4 (2014).

Nikolay Jetchev, Urs Bergmann, and Roland Vollgraf. 2016. Texture Synthesis with Spatial Generative Adversarial Networks. CoRR abs/1611.08207 (2016). arXiv:1611.08207 http://arxiv.org/abs/1611.08207

Ares Lagae, Sylvain Lefebvre, George Drettakis, and Philip Dutré. 2009. Procedural Noise using Sparse Gabor Convolution. ACM Transactions on Graphics (Proceedings of ACM SIGGRAPH 2009) 28, 3 (2009), 54-64.

Gaspard Monge. 1781. Mémoire sur la théorie des déblais et des remblais. Histoire de l'Académie Royale des Sciences de Paris (1781).

Koki Nagano, Graham Fyffe, Oleg Alexander, Jernej Barbiç, Hao Li, Abhijeet Ghosh and Paul E Debevec. 2015. Skin microstructure deformation with displacement map convolution. ACM Transactions on Graphics 34, 4 (2015), 109-1.

Giljoo Nam, Joo Ho Lee, Hongzhi Wu, Diego Gutierrez, and Min H Kim. 2016. Simul taneous acquisition of microscale reflectance and normals. ACM Transactions on Graphics 35, 6 (2016), 185-1.

Ken Perlin. 1985. An Image Synthesizer. In Proceedings of the 12th Annual Conference on Computer Graphics and Interactive Techniques (SIGGRAPH '85). 287-296.

Boris Raymond, Gaël Guennebaud, and Pascal Barla. 2016. Multi-scale Rendering of Scratched Materials Using a Structured SV-BRDF Model. ACM Transactions on Graphics 35, 4 (2016), 57:1-57:11.

Kenneth E Torrance and Ephraim M Sparrow. 1967. Theory for off-specular reflection from roughened surfaces. Fosa 57, 9 (1967), 1105-1114.

Zdravko Velinov, Sebastian Werner, and Matthias B. Hullin. 2018. Real-Time Rendering of Wave-Optical Effects on Scratched Surfaces. Computer Graphics Forum (Proc. of EUROGRAPHICS 2018) 37, 2 (2018).

Bruce Walter, Stephen R Marschner, Hongsong Li, and Kenneth E Torrance. 2007. Microfacet models for refraction through rough surfaces. In Proceedings of the 18th Eurographics conference on Rendering Techniques. 195-206.

Hao Wang. 1961. Proving theorems by pattern recognition - II. The Bell System Technical Journal 40, 1 (1961), 1-41.

Li-Yi Wei and Marc Levoy. 2000. Fast texture synthesis using tree-structured vector quantization. In Proceedings of the 27th annual conference on Computer graphics and interactive techniques. 479-488.

Sebastian Werner, Zdravko Velinov, Wenzel Jakob, and Matthias B. Hullin. 2017. Scratch iridescence: Wave-optical rendering of diffractive surface structure. ACM Transactions on Graphics (Proceedings of SIGGRAPH Asia 2017) 36, 6 (2017), 207:1-207:14.

Ling-Oi Yan, Miloš Hašan, Wenzel Jakob, Jason Lawrence, Steve Marschner, and Ravi Ramamoorthi. 2014. Rendering Glints on High-Resolution Normal-Mapped Specular Surfaces. ACM Transactions on Graphics (Proceedings of SIGGRAPH 2014) 33, 4 (2014).

Ling-Qi Yan, Miloš Hašan, Steve Marschner, and Ravi Ramamoorthi. 2016. PositionNormal Distributions for Efficient Rendering of Specular Microstructure. ACM Transactions on Graphics (Proceedings of SIGGRAPH 2016) 35, 4 (2016).

Ling-Qi Yan, Miloš Hašan, Bruce Walter, Steve Marschner, and Ravi Ramamoorthi. 2018. Rendering Specular Microgeometry with Wave Optics. ACM Transactions on Graphics (Proceedings of SIGGRAPH 2018) 37, 4 (2018).

Qizhi Yu, Fabrice Neyret, Eric Bruneton, and Nicolas Holzschuch. 2011. Lagrangian Texture Advection: Preserving Both Spectrum and Velocity Field. IEEE Transaction on Visualization and Computer Graphics 17, 11 (2011), 1612-1623.

Yang Zhou, Zhen Zhu, Xiang Bai, Dani Lischinski, Daniel Cohen-Or, and Hui Huang 2018. Non-stationary texture synthesis by adversarial expansion. ACM Transactions on Graphics (TOG) 37, 4 (2018), 49.

Tobias Zirr and Anton S Kaplanyan. 2016. Real-time rendering of procedural multiscale materials. In Proceedings of the 20th ACM SIGGRAPH Symposium on Interactive $3 D$ Graphics and Games. ACM, 139-148.

\section{A APPENDIX}

\section{A.1 Pseudo-code}

We present detailed pseudocode of our algorithms in this subsection: building 2D sparse-table (Algorithm 1), using it for range queries (Algorithm 2), and performing top-down traversal (Algorithm 3).

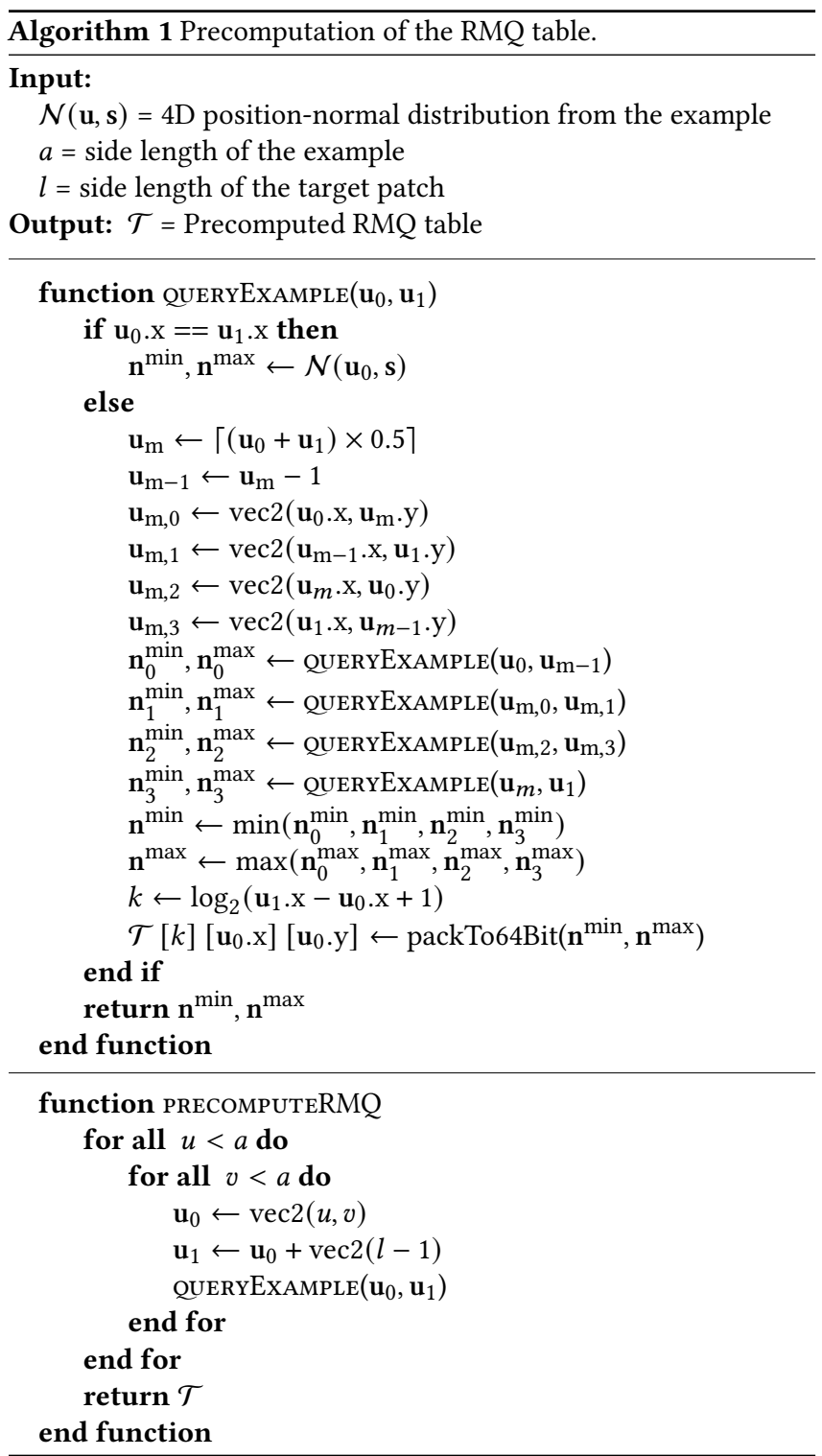

\section{A.2 Jacobian Blending}

We use chain rule to compute the blended Jacobian J. Starting from the individual Jacobians on the four positions located on example patches, we keep track of their individual Jacobians for each function using the chain rule. We consider three different blending methods: linear, variance preserving and histogram preserving blending.

Linear Blending. Since the normals in linear blending go through a set of linear operations (see Equation 4), the formula of the blended Jacobian with chain rule is:

$$
\mathrm{J}_{l}=\sum w_{i} \mathbf{J}_{n}
$$

where $w_{i}$ represents the weight of each example during blending, and $\mathbf{J}_{i}$ is the Jacobian associated with normal $\mathbf{n}$ of the $i_{\text {th }}$ input. 

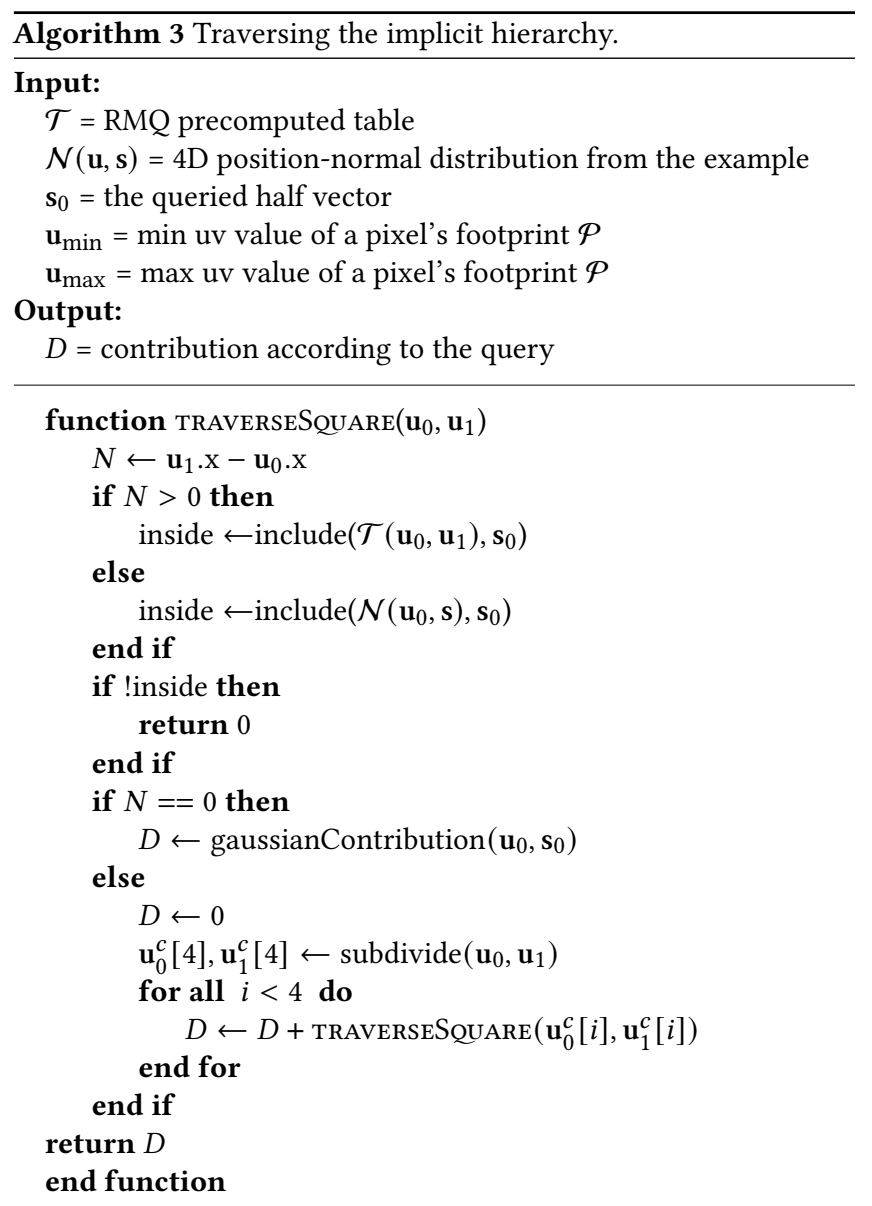

function traverse $\left(\mathbf{u}_{\min }, \mathbf{u}_{\max }\right)$

// Find the minimum square-sized query.

$x \leftarrow \mathbf{u}_{\max } \cdot \mathrm{x}-\mathbf{u}_{\min } \cdot \mathrm{x}$

$y \leftarrow \mathbf{u}_{\max } \cdot \mathrm{y}-\mathbf{u}_{\min } \cdot \mathrm{y}$

$\mathbf{u}_{\min } \leftarrow \mathbf{u}_{\min }$

$\mathbf{u}_{\max }^{\prime} \leftarrow \mathbf{u}_{\min }+2^{\left\lceil\log _{2}(\max (x, y))\right\rceil}$

return TRAVERSESQUARE $\left(\mathbf{u}_{\text {min }}^{\prime}, \mathbf{u}_{\text {max }}^{\prime}\right)$

end function

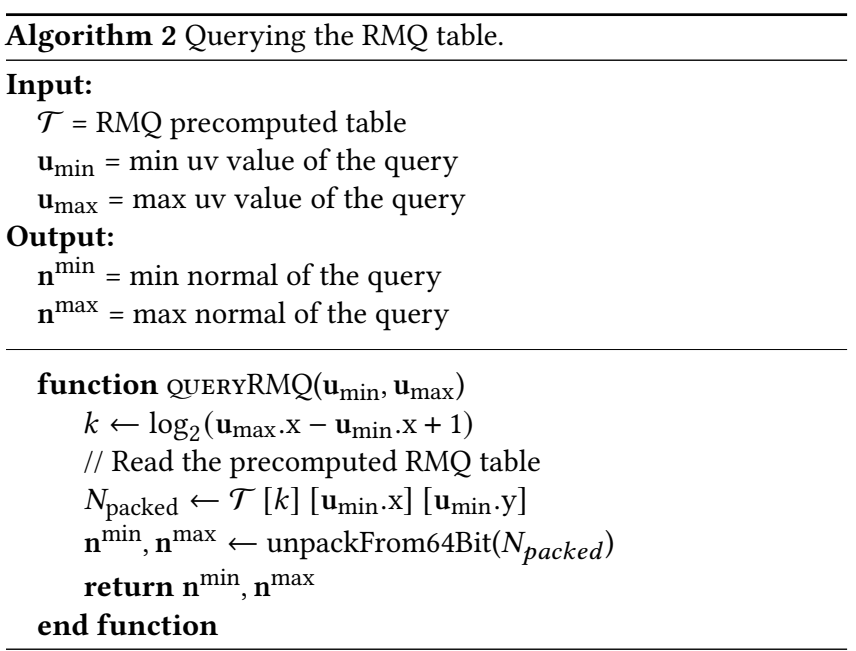

Variance Preserving Blending. In the variance preserving blending, extra linear operations are performed (see Equation 5) after linear blending, resulting in:

$$
\mathrm{J}_{v}=\mathbf{J}_{l} / W
$$

where $W=\sqrt{\sum_{i=1}^{K} w_{i}^{2}}$ is the L2-norm of all the weights.

Histogram Preserving Blending. Additional standardization $(G)$ and inverse standardization $\left(\mathcal{G}^{-1^{\prime}}\right)$ operations are performed in histogram preserving blending, which are non-linear operations. Since these two operations are precomputed in a table, we compute their derivatives along with the precomputed values. During blending, the Jacobian is then computed as follows:

$$
\mathbf{J}_{h}=\mathcal{G}^{-1^{\prime}}\left[\mathcal{G}(\mathbf{n})_{v}\right] \cdot\left[\mathcal{G}^{\prime}(\mathbf{n}) \otimes \mathbf{J}\right]_{v},
$$

where $\mathcal{G}^{-1^{\prime}}$ and $\mathcal{G}^{\prime}$ represent the inverse standardization and standardization derivatives, and they should be diagonal matrices, since we blend the two components of normal, $\mathbf{n}_{x}$ and $\mathbf{n}_{y}$, separately. $\mathcal{G}(\mathbf{n})_{v}$ is the variance blended normal (see Equation 8). $\left[\mathcal{G}^{\prime}(\mathbf{n}) \otimes \mathbf{J}\right]_{v}$ represents performing the standardization operation on the input Jacobian, which is an element-wise matrix multiplication, and then followed by a variance preserving operation. 